\title{
SPATIALLY HETEROCLINIC SOLUTIONS FOR A SEMILINEAR ELLIPTIC P.D.E.*
}

\author{
PAUl H. RaBinowitz ${ }^{1}$
}

\begin{abstract}
This paper uses minimization methods and renormalized functionals to find spatially heteroclinic solutions for some classes of semilinear elliptic partial differential equations
\end{abstract}

Mathematics Subject Classification. 35J20, 35J60, 58E15.

Received January 16, 2002.

\section{INTRODUCTION}

In an earlier paper [6], the equation

$(\mathrm{PDE})-\Delta u=g(x, u), \quad x \in \Omega \subset \mathbb{R}^{n}$

was studied where $\Omega$ is a cylindrical domain in $\mathbb{R}^{n}$, i.e. $\Omega=\mathbb{R} \times \mathcal{D}$ with $\mathcal{D}$ a bounded open set in $\mathbb{R}^{n-1}$ having a smooth boundary. For $x \in \Omega$, set $x=\left(x_{1}, y\right)$ with $x_{1} \in \mathbb{R}$ and $y \in \mathcal{D}$. The function $g$ satisfies

- $\left(g_{1}\right) g \in C^{1}(\Omega \times \mathbb{R}, \mathbb{R})$ and is 1-periodic in $x_{1}$;

- $\left(g_{2}\right) G(x, z)=\int_{0}^{z} g(x, s) \mathrm{d} s$ is 1 -periodic in $z$;

- $\left(g_{3}\right) g$ is even in $x_{1}$.

Letting $\nu(x)$ denote the outward pointing normal to $\partial \Omega$, the boundary condition taken for (PDE) in [6] was (BC) $\frac{\partial u}{\partial \nu}=0, \quad x \in \partial \Omega$.

Let

$$
L(u)=\frac{1}{2}|\nabla u|^{2}-G(x, u)
$$

and $\Omega_{j}=[j, j+1] \times \mathcal{D}$ for $j \in \mathbb{Z}$. By minimizing the functional

$$
I_{0}(u)=\int_{\Omega_{0}} L(u) \mathrm{d} x
$$

over

$$
E_{0}=\left\{u \in W^{1,2}\left(\Omega_{0}\right) \mid u \text { is 1-periodic in } x_{1}\right\},
$$

Keywords and phrases: Spatially heteroclinic solutions, minimization methods, renormalized functional.

* This research was sponsored in part by the National Science Foundation under grant number \#MCS-8110556. Any reproduction for the purposes of the U.S. Government is permitted.

${ }^{1}$ Mathematics Department, University of Wisconsin-Madison, U.S.A.; e-mail: rabinowi@math.wisc.edu

(C) EDP Sciences, SMAI 2002 
it was shown in [6] that hypotheses $\left(g_{1}, g_{2}\right)$ imply there is a classical solution $v$ of (PDE) minimizing $I_{0}$ on $E_{0}$. If in addition $\left(g_{3}\right)$ holds, $v$ is even in $x_{1}$ and

$$
c_{0} \equiv \inf _{E_{0}} I_{0}=I_{0}(v)=\inf _{W^{1,2}\left(\Omega_{0}\right)} I_{0} \equiv c .
$$

Set

$$
\mathcal{M}=\left\{v \in E_{0} \mid I_{0}(v)=c_{0}\right\}
$$

Note that by (PDE) and (BC), $v \in \mathcal{M}$ implies $v+j \in \mathcal{M}$ for all $j \in \mathbb{Z}$. The main result in [6] was if $\left(g_{1}-g_{3}\right)$ hold and $\mathcal{M}$ consists of isolated points, then for any $v \in \mathcal{M}$, there is a $w \in \mathcal{M} \backslash\{v\}$ and a solution, $U$, of (PDE) and (BC) such that $U$ is heteroclinic in $x_{1}$ from $v$ to $w$, i.e. $U(x)-v(x) \rightarrow 0$ as $x_{1} \rightarrow-\infty$ and $U(x)-w(x) \rightarrow 0$ as $x_{1} \rightarrow \infty$. It was also shown that the same result obtains for (PDE) under boundary conditions for different hypotheses on $g$ including a class of water wave problems studied by Kirchgassner [3], Turner [8], and Mielke [4].

The goal of this paper is to improve on [6] in 3 ways. First the structure of $\mathcal{M}$ will be clarified. It will be shown that $\mathcal{M}$ is an ordered set, i.e. $v, w \in \mathcal{M}$ and $v \not \equiv w$ implies $v<w$ or $v>w$. Secondly the condition that $\mathcal{M}$ consists of isolated points will be weakened. Since if $v \in \mathcal{M}$, so is $v+1$, either these two functions are part of a continuum in $\mathcal{M}$, or not. It will be assumed that the latter alternative holds and in particular $v$ and $w$ are adjacent members of $\mathcal{M}$ with $v<w$.

Thirdly, and this is the main contribution of the current paper, condition $\left(g_{3}\right)$ will be dropped. This hypothesis was crucial in [6]. Indeed the approach taken in [6] was to find $U$ as the minimizer of an appropriate functional. The natural functional corresponding to (PDE) is

$$
I(u)=\int_{\Omega} L(u) \mathrm{d} x
$$

but when $c_{0} \neq 0, I(u)$ will not be finite on the class of admissible functions. Therefore a renormalized functional was introduced in [6]. More precisely, set

$\Gamma=\left\{u \in W_{\mathrm{loc}}^{1,2}(\Omega) \mid u(x)-v(x) \rightarrow 0\right.$ as $x_{1} \rightarrow-\infty$ and $u(x)-w(x) \rightarrow 0$ as $x_{1} \rightarrow \infty$ for some $\left.w \in \mathcal{M} \backslash\{v\}\right\}$, and for $u \in \Gamma$, define the renormalized functional for (PDE) via

$$
J(u)=\sum_{j \in \mathbb{Z}} a_{j}(u)
$$

where

$$
a_{j}(u)=\int_{\Omega_{j}} L(u) \mathrm{d} x-c_{0} .
$$

Setting $\tau_{j} u\left(x_{1}, y\right)=u\left(x_{1}-j, y\right)$, it follows that

$$
a_{j}(u)=I_{0}\left(\tau_{-j} u\right)-c_{0} .
$$

Since $\tau_{-j} u \in W^{1,2}\left(\Omega_{0}\right)$, by (1.1),

$$
a_{j}(u) \geq 0
$$

and $J$ is a nonnegative functional. In [6], $U$ was obtained as the minimizer of $J$ on $\Gamma$. Dropping $\left(g_{3}\right)$, it is no longer the case that $c_{0}=c$; in general $c_{0}>c$. Hence (1.3) fails and it is not clear that $J$ is bounded from below on $\Gamma$. Replacing $c_{0}$ by $c$ will not help since then $J$ will be infinite on the natural class of admissible functions. Thus another approach is needed here. For such an approach, we were motivated by some recent work [7] on 
an Allen-Cahn model equation which in turn has antecedents in work of Moser [5] and Bangert [1] on minimal laminations of a torus and of Bosetto and Serra [2] on an ODE problem related to [1]. $\Gamma$ will be replaced by a class of functions asymptotic from $v$ to $w$ and having an additional monotonicity property and $J$ by a related function $J^{*}$. Minimizing $J^{*}$ on $\Gamma^{*}$ will produce the desired heteroclinic joining $v$ and $w$. The proof will be carried out in Section 2 with some details postponed until Section 3. Then in Section 4, it will be indicated how the current approach also applies to the water wave problem of Kirchgässner [3].

\section{The MAIN Results}

In this section, our main results will be formulated and proved. As was noted in Section 1, it was shown in [6] that $\mathcal{M} \neq \phi$ and the elements of $\mathcal{M}$ are classical solutions of (PDE) and (BC) which are 1-periodic in $x_{1}$. The first result uses the variational structure of (PDE) and the Maximum Principle to say more about $\mathcal{M}$.

Proposition 2.1. If $g$ satisfies $\left(g_{1}, g_{2}\right), \mathcal{M}$ is an ordered set.

Proof. An argument essentially due to Moser [5] will be employed. Suppose $v \neq w \in \mathcal{M}$ and $v$ and $w$ are not ordered. Set $\varphi=\max (v, w)$ and $\psi=\min (v, w)$. Then $\varphi, \psi \in E_{0}$. For functions $a, b$ on $\Omega_{0}$,

$$
\{a \leq b\} \equiv\left\{x \in \Omega_{0} \mid a(x) \leq b(x)\right\}
$$

and $\{a<b\}$, the same with strict inequality. Hence

$$
2 c_{0} \leq I_{0}(\varphi)+I_{0}(\psi)=\int_{\{w \geq v\}} L(w) \mathrm{d} x+\int_{\{v>w\}} L(v) \mathrm{d} x+\int_{\{w \geq v\}} L(v) \mathrm{d} x+\int_{\{v>w\}} L(w) \mathrm{d} x=2 c_{0} .
$$

Therefore $\varphi, \psi \in \mathcal{M}$ and by [6] are classical solutions of (PDE) and (BC). Since $v$ and $w$ are not ordered, without loss of generality there are points $\underline{x}, \bar{x} \in \Omega_{0}$ such that $v(\bar{x})>w(\bar{x})$ and $v(\underline{x})=w(\underline{x})$. Consider $\chi \equiv \varphi-w$. Then $\chi \geq 0$ in $\bar{\Omega}_{0}$ and $\chi(\bar{x})>0$. Observe that $\chi$ satisfies a linear elliptic partial differential equation in $\Omega_{0}$ :

$$
\left\{\begin{aligned}
-\Delta \chi & =a(x) \chi, & & x \in \Omega_{0} \\
\frac{\partial \chi}{\partial \nu} & =0 & & x \in[0,1] \times \partial \mathcal{D}
\end{aligned}\right.
$$

where

$$
\left\{\begin{aligned}
a(x) & =\frac{g(x, \varphi(x))-g(x, w(x))}{\varphi(x)-w(x)} & & \text { if } \varphi(x)>w(x) \\
& =g_{u}(x, \varphi(x)) & & \text { if } \varphi(x)=w(x)
\end{aligned}\right.
$$

Writing $a(x)=a^{+}(x)-a^{-}(x)$ where

$$
a^{+}=\max (a, 0) ; \quad a^{-}=\max (a, 0)
$$

equation (2.3) leads to

$$
\begin{cases}-\Delta \chi+a^{-} \chi=a^{+} \chi \geq 0, & \text { if } x \in \Omega_{0} \\ \frac{\partial \chi}{\partial \nu}=0, & \text { if } x \in[0,1] \times \partial \mathcal{D}\end{cases}
$$


By the Maximum Principle, $\chi$ cannot have an interior minimum in $\Omega_{0}$ unless $\chi \equiv$ constant which is not the case because $v$ and $w$ are not ordered. Since $\chi(\underline{x})=0, \underline{x} \in[0,1] \times \partial \mathcal{D}$. But then by the Maximum Principle,

$$
\frac{\partial \chi}{\partial \nu}(\underline{x})<0
$$

contrary to (2.3). Thus $v$ and $w$ are ordered.

To continue, as was mentioned in the introduction, $\mathcal{M}$ may be a connected set. E.g. if $g \equiv 0, \mathcal{M}=\mathbb{R}$. For the existence argument that will be given shortly, it is necessary that $\mathcal{M}$ is not connected. Thus for what follows, it will be assumed that:

$(*)$ there are adjacent members $v<w$ of $\mathcal{M}$.

Next it will be shown that $(*)$ implies there is a solution of (PDE) and (BC) heteroclinic in $x_{1}$ from $v$ to $w$ and similarly from $w$ to $v$. This will be done by minimizing a variant of the renormalized functional $J$ of (1.2) over an appropriate class of sets, $\Gamma^{*}$. To motivate the choice of $\Gamma^{*}$, proceeding formally, suppose that $J$ has a minimizer in the class of $W_{\text {loc }}^{1,2}(\mathbb{R} \times \mathcal{D})$ functions heteroclinic in $x_{1}$ from $v$ to $w$. Suppose further the minimizer is a classical solution of (PDE) and (BC). Then repeating the proof of Proposition 2.1 for this new setting shows $\mathcal{M}^{*}$, the set of minimizers of $J$, is an ordered set. Moreover if $U \in \mathcal{M}^{*},\left(g_{1}\right)$ implies $J\left(\tau_{j} U\right)=J(U)$ for all $j \in \mathbb{Z}$. Hence $\tau_{j} U \in \mathcal{M}^{*}$. The ordering property of $\mathcal{M}^{*}$ then implies $\tau_{-1} U>U$, i.e. $U$ has a monotonicity property. This formal argument will be exploited by building the monotonicity into the class of functions $\Gamma^{*}$ and it will be crucial for what follows. Define

$\Gamma^{*}=\left\{u \in W_{\mathrm{loc}}^{1,2}(\mathbb{R} \times \mathcal{D}) \mid v \leq u \leq \tau_{-1} u \leq w\right.$, and $\left.\tau_{-j} u\right|_{\Omega_{0}} \rightarrow v($ resp. $w)$ in $L^{2}\left(\Omega_{0}\right)$ as $j \rightarrow-\infty($ resp. $\left.\infty)\right\}$

To introduce the analogue of $J$ of (1.2) that will be employed here, let $m, n \in \mathbb{Z}$ with $m \leq n$. For $u \in \Gamma^{*}$, set

$$
J_{m, n}(u)=\sum_{m}^{n} a_{j}(u)
$$

and define

$$
J^{*}(u)=\underline{\lim }_{m \rightarrow-\infty} J_{m, 0}(u)+\underline{\lim }_{n \rightarrow \infty} J_{1, n}(u) .
$$

Set

$$
c^{*}=\inf _{u \in \Gamma^{*}} J^{*}(u)
$$

The main result of this section is:

Theorem 2.2. Let $g$ satisfy $\left(g_{1}, g_{2}\right)$ and let $(*)$ hold. Then there is a $U \in \Gamma^{*}$ such that $J^{*}(U)=c^{*}$. Moreover $U$ is a classical solution of $(P D E)$ and $(B C)$.

The proof of Theorem 2.2 is divided into several steps. So as not to delay the exposition, the details of some of the steps will be postponed until Section 3. Although the setting is different, the structure of the argument is very close to that of [7] which we will strongly follow. 
To begin, note that $c^{*}<\infty$. Indeed if

$$
\begin{aligned}
\varphi(x) & =v, \quad x_{1} \leq 0 \\
& =x_{1} w+\left(1-x_{1}\right) v, \quad 0 \leq x_{1} \leq 1 \\
& =w, \quad 1 \leq x_{1}
\end{aligned}
$$

then $\varphi \in \Gamma^{*}$ and $c^{*} \leq J^{*}(\varphi)<\infty$.

Next it will be shown that $J_{m, n}$ is bounded from below on $\Gamma^{*}$. This requires a preliminary result.

Lemma 2.3. Let $n \in \mathbb{N}$ and $\Gamma_{n}=\left\{u \in W_{\text {loc }}^{1,2}(\mathbb{R} \times \mathcal{D}) \mid u\right.$ is $n$ periodic in $\left.x_{1}\right\}$. If

$$
c_{n}=\inf _{u \in \Gamma_{n}} \int_{0}^{n} \int_{\mathcal{D}} L(u) \mathrm{d} x .
$$

Then $c_{n}=n c_{0}$ and is achieved by $v \in \mathcal{M}$.

Proof. The existence of a minimizer, $v$ of (2.8) follows as in [6] for the case of $n=1$. It remains to prove that

$$
\int_{0}^{n} \int_{\mathcal{D}} L(v) \mathrm{d} x=n c_{0} .
$$

By $\left(g_{1}\right), \tau_{-1} v$ is also a minimizer of (2.8) and the proof of Proposition 2.1 shows that the set of minimizers of (2.8) is ordered. Therefore either (i) $\tau_{-1} v \equiv v$, (ii) $\tau_{-1} v<v$, or (iii) $\tau_{-1} v>v$. If e.g. (ii) is valid,

$$
v\left(x_{1}+1, y\right)<v\left(x_{1}, y\right)<v\left(x_{1}-(n-1), y\right)=v\left(x_{1}+1, y\right),
$$

a contradiction. Likewise (iii) cannot hold. Thus (i) is valid, i.e. $v$ is 1 -periodic in $x_{1}, c_{n}=n c_{0}$, and the result is proved.

Now it follows that $J_{m, n}$ is bounded from below:

Proposition 2.4. There is a constant $K>0$ such that for all $u \in \Gamma^{*}$ and $m \leq n$,

$$
J_{m, n}(u) \geq-K
$$

The proof of Proposition 2.4 will be given in Section 3. The proposition implies an upper bound for $J_{m, n}(u)$ :

Lemma 2.5. For all $m \leq n$ and $u \in \Gamma^{*}$,

$$
J_{m, n}(u) \leq J(u)+2 K
$$

Proof. By (2.11),

$$
J(u)=\underline{\lim }_{i \rightarrow-\infty} J_{i, 0}(u)+\underline{\lim }_{i \rightarrow \infty} J_{1, i}(u)=J_{m, n}(u)+\underline{\lim }_{i \rightarrow-\infty} J_{i, m-1}(u)+\underline{\lim }_{i \rightarrow \infty} J_{n+1, i}(u) \geq J_{m, n}(u)-2 K
$$

from which (2.12) follows. 
The next step in the proof is to show that the finiteness of $J^{*}(u)$ implies some strong asymptotic properties for $u$ :

Proposition 2.6. Let $u \in \Gamma^{*}$ and $J^{*}(u)<\infty$. Then

$$
J^{*}(u)=\lim _{\substack{m \rightarrow-\infty \\ n \rightarrow \infty}} J_{m, n}(u),
$$

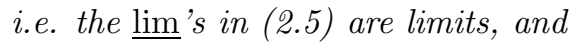

$$
\begin{gathered}
\lim _{m \rightarrow-\infty}\|u-v\|_{W^{1,2}\left(\Omega_{m}\right)}=0 \\
\lim _{n \rightarrow \infty}\|u-w\|_{W^{1,2}\left(\Omega_{n}\right)}=0 .
\end{gathered}
$$

The proof of this proposition will be given in Section 3.

With the aid of the above preliminaries, the function $U$ of Theorem 2.2 can be obtained. Let $\left(u_{k}\right)$ be a minimizing sequence for (2.6). Then there is an $M>0$ such that

$$
J\left(u_{k}\right) \leq M
$$

for all $k \in \mathbb{N}$. Since $u \in \Gamma^{*}$ implies $\tau_{j} u \in \Gamma^{*}$ for all $j \in \mathbb{Z}$, and $J^{*}\left(\tau_{j} u\right)=J^{*}(u)$, the sequence $\left(u_{k}\right)$ can be normalized by requiring that

$$
\left\{\begin{array}{l}
\int_{\Omega_{-1}}\left(u_{k}-v\right) \mathrm{d} x \leq \frac{1}{2} \int_{\Omega_{0}}(w-v) \mathrm{d} x \\
\int_{\Omega_{0}}\left(u_{k}-v\right) \mathrm{d} x \geq \frac{1}{2} \int_{\Omega_{0}}(w-v) \mathrm{d} x
\end{array}\right.
$$

Note that since $u_{k} \leq \tau_{-1} u_{k}$,

$$
\left\{\begin{array}{l}
\int_{\Omega_{j}}\left(u_{k}-v\right) \mathrm{d} x \leq \frac{1}{2} \int_{\Omega_{0}}(w-v) \mathrm{d} x, \quad j \leq-1 \\
\int_{\Omega_{j}}\left(u_{k}-v\right) \mathrm{d} x \geq \frac{1}{2} \int_{\Omega_{0}}(w-v) \mathrm{d} x, \quad j \geq 0 .
\end{array}\right.
$$

By (2.12) and (2.16),

$$
\frac{1}{2} \int_{m}^{n+1} \int_{\mathcal{D}}\left|\nabla u_{k}\right|^{2} \mathrm{~d} x \leq \int_{m}^{n+1} \int_{\mathcal{D}} G\left(x, u_{k}\right) \mathrm{d} x+(n+1-m) c_{0}+M+2 K .
$$

Thus (2.19) coupled with the $L^{\infty}$ bounds for $u_{k}$ imply $\left(u_{k}\right)$ is bounded in $W_{\text {loc }}^{1,2}(\Omega)$. Hence there is a $U \in W_{\text {loc }}^{1,2}(\Omega)$ such that along a subsequence, $u_{k} \rightarrow U$ weakly in $W_{\text {loc }}^{1,2}$, strongly in $L_{\text {loc }}^{2}$, and pointwise a.e. Thus

$$
v \leq U \leq \tau_{-1} U \leq w
$$

and $U$ satisfies (2.18). By weak lower semicontinuity and (2.12) again,

$$
J_{m, n}(U) \leq \underline{\lim }_{k \rightarrow \infty} J_{m, n}\left(u_{k}\right) \leq M+2 K
$$


for all $m \leq n$ in $\mathbb{Z}$. Hence

$$
J^{*}(U) \leq M+2 K<\infty .
$$

To show that $U \in \Gamma^{*}$, it remains to prove that $U$ is heteroclinic from $v$ to $w$. Towards that end, for $x \in \Omega_{0}$, set $V_{i}=\tau_{i} U$. Then $\left(V_{i}\right)$ is a monotone nonincreasing sequence as $i \rightarrow \infty$ and $V_{i}$ is bounded in $W^{1,2}\left(\Omega_{0}\right)$ via $(2.21)$ with $m=n=-i$. Therefore there is a $V \in W^{1,2}\left(\Omega_{0}\right)$ such that $V_{i} \rightarrow V$ weakly in $W^{1,2}\left(\Omega_{0}\right)$ and strongly in $L^{2}\left(\Omega_{0}\right)$ as $i \rightarrow \infty$. Since

$$
\lim _{i \rightarrow \infty} V_{i}=V=\lim _{i \rightarrow \infty} \tau_{1} V_{i}=\tau_{1} V
$$

$V \in E_{0}$. We claim $V \in \mathcal{M}$. If not, there is a $\rho>0$ such that

$$
I_{0}(V)>c_{0}+\rho .
$$

Since

$$
I_{0}(V) \leq \varliminf_{i \rightarrow \infty} I_{0}\left(V_{i}\right)
$$

for all large $i$,

$$
I_{0}\left(V_{i}\right)=I_{0}\left(\tau_{i} U\right) \geq c_{0}+\rho / 2
$$

But then

$$
J_{i, 0}(U) \rightarrow \infty
$$

as $i \rightarrow-\infty$, contrary to (2.21). Thus $V \in \mathcal{M}$ and by (2.20) lies between $v$ and $w$. Moreover by (2.18) with $u_{k}$ replaced by $U$, letting $j \rightarrow-\infty$ shows

$$
\int_{\Omega_{0}}(V-v) \mathrm{d} x \leq \frac{1}{2} \int_{\Omega_{0}}(w-v) \mathrm{d} x .
$$

Consequently by condition (*), $V=v$. Similarly $V_{i} \rightarrow w$ as $i \rightarrow-\infty$ and $U \in \Gamma^{*}$.

The next step in our argument is:

Proposition 2.7. $J^{*}(U)=c^{*}$.

The proof of this proposition will be given in Section 3.

The final step in the proof of Theorem 2.2 is to verify that $U$ is a classical solution of (PDE) and (BC). This is a consequence of local minimization properties that the global minimizer, $U$ of (2.6) possesses. To be more precise, let $B_{r}(z)$ denote an open ball of radius $r$ about $z \in \mathbb{R}^{n}$. Suppose $B_{2 r}(z) \subset \Omega$. Set

$$
A_{r}(z)=\left\{u \in W^{1,2}\left(B_{2 r}(z)\right) \mid u=U \text { in } B_{2 r}(z) \backslash B_{r}(z)\right\} .
$$

For $u \in A_{r}(z)$, define

$$
F_{r}(u)=\int_{B_{r}(z)} L(u) \mathrm{d} x
$$

The local minimization property for interior points is:

Proposition 2.8. For each $z \in \Omega$ and $0<r<\frac{1}{2}$ such that $B_{2 r}(z) \subset \Omega, U$ minimizes $F_{r}$ over $A_{r}(z)$. 
This result will also be proved in Section 3. Since

$$
F_{r}(U)=\inf _{u \in A_{r}(z)} F_{r}(u)
$$

and, by standard elliptic regularity arguments, any minimizer of $F_{r}$ on $A_{r}(z)$ is a classical solution of (PDE) in $B_{r}(z), U$ is a classical solution of $(\mathrm{PDE})$ in $\Omega$, and is even in $C_{\text {loc }}^{2, \alpha}(\Omega)$ for any $\alpha \in(0,1)$.

It remains only to show that $U$ is smooth up to $\partial \Omega$ and (BC) holds. This follows as above by a slight variant of Proposition 2.8. Let $z \in \partial \Omega$ and $r>0$. Consider

$$
\hat{F}_{r}(u)=\int_{B_{r}(z) \cap \Omega} L(u) \mathrm{d} x
$$

on

$$
\hat{A}_{r}(z)=\left\{u \in W^{1,1}\left(B_{2 r}(z) \cap \Omega\right) \mid u=U \text { in }\left(B_{2 r}(z) \backslash B_{r}(z)\right) \cap \Omega\right\} .
$$

The analogue of Proposition 2.8 here is:

Proposition 2.9. For each $z \in \partial \Omega$ and $0<r<\frac{1}{2}$, $U$ minimizes $\hat{F}_{r}$ on $\hat{A}_{r}(z)$.

The proof of this result will be sketched after that of Proposition 2.8 .

By the regularity of $U$ given by Proposition $2.9, U$ is a $C^{2, \alpha}$ function up to $\partial \Omega$ and $\frac{\partial U}{\partial \nu}=0$ on $\partial \Omega$. The proof of Theorem 2.2 is complete.

\section{Proofs of the technical Results}

This section is devoted to the proof of Propositions 2.4, and 2.6-2.9.

Proof of Proposition 2.4. Define

$$
\varphi(x)= \begin{cases}\left(x_{1}-m\right) u(x)+\left(m+1-x_{1}\right) v(x), & m \leq x_{1} \leq m+1, \\ u(x), & m+1 \leq x_{1} \leq n \\ \left(x_{1}-n\right) v(x)+\left(n+1-x_{1}\right) u(x), & n \leq x_{1} \leq n+1\end{cases}
$$

and continue $\varphi$ to $\mathbb{R} \times \mathcal{D}$ as an $n-m+1$ periodic function. Then by Lemma 2.3,

$$
J_{m, n}(\varphi) \geq 0
$$

By (3.1),

$$
\begin{aligned}
\int_{\Omega_{m}} L(\varphi) \mathrm{d} x= & \int_{\Omega_{m}}\left[\frac{1}{2}\left|\left(x_{1}-m\right) \nabla u+\left(m+1-x_{1}\right) \nabla v\right|^{2}+\left(x_{1}-m\right) u_{x_{1}}+\left(m+1-x_{1}\right) v_{x_{1}}(u-v)\right. \\
& \left.+\frac{1}{2}(u-v)^{2}+G(x, \varphi)\right] \mathrm{d} x .
\end{aligned}
$$

The terms in (3.3) will be estimated separately. First,

$$
\left|\left(x_{1}-m\right) \nabla u+\left(m+1-x_{1}\right) \nabla v\right|^{2} \leq\left[\left(x_{1}-m\right)^{2}+\left(m+1-x_{1}\right)^{2}\right]\left(|\nabla u|^{2}+|\nabla v|^{2}\right) \leq|\nabla u|^{2}+|\nabla v|^{2} .
$$

Next

$$
T=\left(x_{1}-m\right) u_{x_{1}}+\left(m+1-x_{1}\right) v_{x_{1}}(u-v)=\left(x_{1}-m\right) \frac{\partial}{\partial x_{1}} \frac{(u-v)^{2}}{2}+(u-v) v_{x_{1}} .
$$


Therefore, since $u-v \leq w-v \leq 1$,

$$
\begin{aligned}
\int_{\Omega_{m}} T \mathrm{~d} x & \leq\left.\int_{\mathcal{D}}\left(x_{1}-m\right) \frac{(u-v)^{2}}{2}\right|_{m} ^{m+1} \mathrm{~d} y+\frac{1}{2} \int_{\Omega_{m}}(u-v)^{2} \mathrm{~d} x+\frac{1}{2} \int_{\Omega_{m}} v_{x_{1}}^{2} \mathrm{~d} x \\
& \leq \frac{1}{2}|\mathcal{D}|+\frac{1}{2}|\mathcal{D}|+\frac{1}{2} \int_{\Omega_{m}} v_{x_{1}}^{2} \equiv K_{1}
\end{aligned}
$$

where $|\mathcal{D}|$ denotes the measure of $\mathcal{D}$. The last integral in (3.3) can be estimated via

$$
\begin{aligned}
\int_{\Omega_{m}} G(x, \varphi) \mathrm{d} x & =\int_{\Omega_{m}}(G(x, \varphi)-G(x, v)+G(x, v)) \mathrm{d} x \leq M_{1} \int_{\Omega_{m}}(\varphi-v) \mathrm{d} x+\int_{\Omega_{m}} G(x, v) \mathrm{d} x \\
& \leq M_{1}|\mathcal{D}|+\int_{\Omega_{m}} G(x, v) \mathrm{d} x \equiv K_{2}
\end{aligned}
$$

where $M_{1}$ is a Lipschitz constant for $G(x, z)$ on $\bar{\Omega} \times[\min v, \max w]$. Combining (3.3-3.7) gives

$$
\int_{\Omega_{m}} L(\varphi) \mathrm{d} x \leq \int_{\Omega_{m}} \frac{1}{2}\left(|\nabla u|^{2}+|\nabla v|^{2}\right) \mathrm{d} x+K_{1}+K_{2} \leq \int_{\Omega_{m}} L(u) \mathrm{d} x+\left|\int_{\Omega_{m}} G(x, u) \mathrm{d} x\right|+K_{3} .
$$

As in (3.7),

$$
\left|\int_{\Omega_{m}} G(x, u) \mathrm{d} x\right| \leq M_{1} \int_{\Omega_{m}}(u-v) \mathrm{d} x+\left|\int_{\Omega_{m}} G(x, v) \mathrm{d} x\right|
$$

Hence

$$
a_{m}(\varphi) \leq a_{m}(u)+K_{4}
$$

Combining $(3.2,3.10)$, and a similar estimate for $a_{n}(\varphi)$ yields

$$
J_{m, n}(u)=J_{m, n}(\varphi)+a_{m}(u)-a_{m}(\varphi)+a_{n}(u)-a_{n}(\varphi) \geq-K
$$

and Proposition 2.4 is proved.

Proof of Proposition 2.6. Set $u_{k}=\tau_{k} u$. As following (2.22), $u_{k} \rightarrow v$ (resp. $w$ ) as $k \rightarrow \infty$ (resp. $-\infty$ ) weakly in $W^{1,2}\left(\Omega_{0}\right)$ and strongly in $L^{2}\left(\Omega_{0}\right)$. Moreover as in $(2.24-2.27)$,

$$
\varliminf_{|k| \rightarrow \infty} I_{0}\left(u_{k}\right)=c_{0}
$$

Now (3.12) will be used to prove (2.13-2.15). Choose a sequence $k_{i} \rightarrow \infty$ such that

$$
\lim _{i \rightarrow \infty} I_{0}\left(u_{k_{i}}\right)=c_{0}
$$

We claim

$$
\lim _{i \rightarrow \infty}\left\|u_{k_{i}}-v\right\|_{W^{1,2}\left(\Omega_{0}\right)}=0 .
$$

By the convergence already established for $u_{k_{i}}$, it suffices to show

$$
\lim _{i \rightarrow \infty}\left\|\nabla u_{k_{i}}-\nabla v\right\|_{L^{2}\left(\Omega_{0}\right)}=0
$$


To verify (3.15), note first that

$$
\lim _{i \rightarrow \infty}\left\|\nabla u_{k_{i}}\right\|_{L^{2}\left(\Omega_{0}\right)}=\|\nabla v\|_{L^{2}\left(\Omega_{0}\right)}
$$

for if not, by the weak lower semicontinuity of $\|\cdot\|_{L^{2}\left(\Omega_{0}\right)}$, there is a $\delta>0$ such that

$$
\frac{1}{2}\|\nabla v\|_{L^{2}\left(\Omega_{0}\right)}^{2}+\delta \leq \frac{1}{2} \lim _{i \rightarrow \infty}\left\|\nabla u_{\ell_{i}}\right\|_{L^{2}\left(\Omega_{0}\right)}^{2}
$$

where $\ell_{i}$ is a subsequence of $k_{i}$. But then

$$
I_{0}(v)+\delta \leq \lim _{i \rightarrow \infty} \frac{1}{2}\left\|\nabla u_{\ell_{i}}\right\|_{L^{2}\left(\Omega_{0}\right)}^{2}+\underline{\lim }_{i \rightarrow \infty} \int_{\Omega_{0}} G\left(x, u_{\ell_{i}}\right) \mathrm{d} x=\underline{\lim }_{i \rightarrow \infty} I_{0}\left(u_{\ell_{i}}\right)=c_{0}
$$

contrary to (3.13). Now as $i \rightarrow \infty$,

$$
\left\|\nabla u_{k_{i}}-\nabla v\right\|_{L^{2}\left(\Omega_{0}\right)}^{2}=\left\|\nabla u_{k_{i}}\right\|_{L^{2}\left(\Omega_{0}\right)}^{2}+\|\nabla v\|_{L^{2}\left(\Omega_{0}\right)}^{2}-2 \int_{\Omega_{0}} \nabla u_{k_{i}} \cdot \nabla v \mathrm{~d} x \rightarrow 0
$$

via (3.16) and the weak convergence of in $L^{2}(\Omega)$ of $\nabla u_{k_{i}}$ to $\nabla v$. Thus (3.15) holds.

Next (3.12) will be strengthened to show that

$$
\lim _{k \rightarrow \infty} I_{0}\left(u_{k}\right)=c_{0}
$$

Then the arguments in (3.13-3.18) show (2.14) holds and the analogue of (3.19) for $k \rightarrow-\infty$ yields (2.15). To get (3.19), it will be shown that each of the following limits exist:

$$
\text { (i) } \lim _{k \rightarrow-\infty} J_{k, 0}(u) ; \quad \text { (ii) } \lim _{k \rightarrow \infty} J_{1, k}(u) \text {. }
$$

Then (3.20) (i) implies $a_{k}(u) \rightarrow 0$ as $k \rightarrow-\infty$ and therefore (3.19) is valid.

To prove (3.20), (i), set

$$
\mathcal{N}^{-}=\left\{n \in-\mathbb{N} \mid a_{n}(u) \leq 0\right\} .
$$

Suppose first that $\mathcal{N}^{-}$is finite. Then $J_{n, 0}(u)$ is an increasing sequence which is bounded from above as $n \rightarrow-\infty$ via (2.12). Thus (3.20) (i) is verified. On the other hand if $\mathcal{N}^{-}$is an infinite set, the sequence $\left(n_{i}\right)$ it represents must satisfy (3.13) so by (3.14) as $i \rightarrow \infty$,

$$
\left\|u_{n_{i}}-v\right\|_{W^{1,2}\left(\Omega_{0}\right)} \rightarrow 0 .
$$

If the limit (3.20) (i) does not exist, then $\ell^{-}<\ell^{+}$where

$$
\ell^{-} \equiv \underline{\lim }_{n \rightarrow-\infty} J_{n, 0}(u) ; \ell^{+}=\varlimsup_{n \rightarrow-\infty} J_{n, 0}(u) .
$$

It will be shown that $\ell^{-}<\ell^{+}$is not possible.

Choose $\epsilon$ satisfying

$$
0<\epsilon<\frac{\ell^{+}-\ell^{-}}{3} \text {. }
$$


By (3.23), there are sequences $\left(p_{k}\right),\left(q_{k}\right) \subset-\mathbb{N}$ with $p_{k}, q_{k} \rightarrow-\infty$ as $k \rightarrow \infty$ and such that $q_{k}>p_{k}>q_{k+1}$, and

$$
J_{p_{k}, 0}(u) \rightarrow \ell^{-} ; \quad J_{q_{k}, 0}(u) \rightarrow \ell^{+}
$$

as $k \rightarrow \infty$. Hence for $k$ large,

$$
J_{p_{k}, 0}(u) \leq \ell^{-}+\epsilon<\ell^{+}-\epsilon \leq J_{q_{k}, 0}(u)
$$

Set $s_{k}$ the largest $q \in \mathcal{N}^{-}$such that $q<q_{k}$ and set $r_{k}$ the smallest $p \in \mathcal{N}^{-}$such that $p \geq p_{k}$. As a function of $t, J_{s_{k}+t, 0}(u)$ is increasing in $\left(0, q_{k}-1-s_{k}\right]$ while $J_{p_{k}+t, 0}(u)$ decreases in $\left[0, r_{k}-p_{k}\right]$. Therefore

$$
J_{r_{k}, 0}(u) \leq \ell^{-}+\epsilon<\ell^{+}-\epsilon \leq J_{s_{k}, 0}(u)
$$

and

$$
J_{r_{k}, s_{k}-1}(u)=J_{r_{k}, 0}(u)-J_{s_{k}, 0}(u) \leq-\left(\ell^{+}-\ell^{-}\right)+2 \epsilon
$$

Now by (3.22), $u$ is close to $v$ in $W^{1.2}\left(\Omega_{\ell}\right)$ for $\ell=s_{k}$ and $\ell=r_{k}$ and $k$ large. This allows us to modify $u$ in $\Omega_{\ell}$ so as to produce a function, $\psi$, which equals $v$ in $\left[r_{k}, r_{k}+\frac{1}{2}\right]$ and in $\left[s_{k}+\frac{1}{2}, s_{k}+1\right]$. Hence $\psi$ extends to $\Omega$ as a $s_{k}-r_{k}+1$ periodic function in $x_{1}$ so

$$
J_{r_{k}, s_{k}}(\psi) \geq 0
$$

via Lemma 2.3. More precisely, $\psi$ is given by

$$
\left\{\begin{aligned}
\psi(x) & =v(x), \quad r_{k} \leq x_{1} \leq r_{k}+\frac{1}{2} \\
& =2\left(x_{1}-\left(r_{k}+\frac{1}{2}\right) u(x)+2\left(\left(r_{k}+1\right)-x_{1}\right) v(x), \quad r_{k}+\frac{1}{2} \leq x_{1} \leq r_{k}+1\right. \\
& =u(x), \quad r_{k}+1 \leq x_{1} \leq s_{k} \\
& =2\left(x_{1}-s_{k}\right) v(x)+2\left(s_{k}+\frac{1}{2}-x_{1}\right) u(x), \quad s_{k} \leq x_{1} \leq s_{k}+\frac{1}{2} \\
& =v(x), \quad s_{k}+\frac{1}{2} \leq x_{1} \leq s_{k}+1 .
\end{aligned}\right.
$$

Then for $k$ large enough,

$$
\left|a_{r_{k}}(u)\right|+\left|a_{r_{k}}(\psi)\right|+\left|a_{s_{k}}(u)\right|+\left|a_{s_{k}}(\psi)\right|<\epsilon
$$

via (3.22) and straightforward estimates. Consequently by (3.29) and (3.31),

$$
J_{r_{k}, s_{k-1}}(u)=J_{r_{k}, s_{k}}(\psi)-a_{r_{k}}(\psi)+a_{r_{k}}(u)-a_{s_{k}}(\psi) \geq-\epsilon .
$$

Combining (3.32) and (3.28) shows

$$
\ell^{+}-\ell^{-} \leq 3 \epsilon
$$

contrary to (3.24). Thus (3.20) (i) has been verified and (3.20) (ii) follows similarly. The proof of Proposition 2.6 is complete. 
Proof of Proposition 2.34. This proof has some elements in common with that of Proposition 2.6. Suppose $J^{*}(U) \neq c^{*}$. Then since $U \in \Gamma^{*}$, by $(2.6)$, there is a $\sigma>0$ such that

$$
J^{*}(U)=c^{*}+9 \sigma .
$$

By Proposition 2.6,

$$
J^{*}(U)=\lim _{n \rightarrow \infty} J_{-n, n}(U) .
$$

Hence there is an $n_{0} \in \mathbb{N}$ such that if $n \geq n_{0}$,

$$
J_{-n, n}(U) \geq c^{*}+8 \sigma .
$$

By the weak lower semicontinuity of $J_{-n, n}$ and (3.36), there is a $k_{0}(n) \in \mathbb{N}$ such that if $k \geq k_{0}$,

$$
J_{-n, n}\left(u_{k}\right) \geq J_{-n, n}(U)-\sigma \geq c^{*}+7 \sigma .
$$

Moreover since $\left(u_{k}\right)$ is a minimizing sequence for (2.6), for $k$ large,

$$
J^{*}\left(u_{k}\right) \leq c^{*}+\sigma .
$$

Let $T_{n}(u)$ denote the tail of $J^{*}(u)$, i.e.

$$
T_{n}(u)=J_{-\infty,-n-1}(u)+J_{n+1, \infty}(u) \equiv T_{n}^{-}(u)+T_{n}^{+}(u) .
$$

Therefore by $(3.37,3.38)$ for $n \geq n_{0}$ and $k$ large,

$$
c^{*}+\sigma \geq J_{-n, n}\left(u_{k}\right)+T_{n}\left(u_{k}\right) \geq c^{*}+7 \sigma+T_{n}\left(u_{k}\right)
$$

or

$$
T_{n}\left(u_{k}\right) \leq-6 \sigma .
$$

Next it will be shown that (3.39) is not possible, i.e. the tail of $u_{k}$ is uniformly small provided that $n$ and $k$ are sufficiently large. It suffices to show that

$$
T_{n}^{i}\left(u_{k}\right)>-3 \sigma, \quad i=+,-.
$$

The - case will be verified; the + case is handled in the same way.

Let $\delta>0$, free for the moment. Since $U \in \Gamma^{*}$ and $J^{*}(U)<\infty$, by (2.14) for $n=n(\delta)$ large enough,

$$
\|U-v\|_{W^{1,2}\left(\Omega_{-n-1}\right)} \leq \delta
$$

We claim

$$
\left\|u_{k}-U\right\|_{W^{1,2}\left(\Omega_{-n-1}\right)} \leq \delta
$$

for $n(\delta)$ large and appropriate $k$. Assuming (3.42) for now, by $(3.41,3.42)$,

$$
\left\|u_{k}-v\right\|_{W^{1,2}\left(\Omega_{-n-1}\right)} \leq 2 \delta .
$$


Let $N \in \mathbb{N}$ with $N>n+1$ and let $\psi$ be as in (3.30) with $u$ replaced by $u_{k}, r$ by $N$, and $s$ by $-n-1$. Then (3.29) becomes

$$
J_{-N,-n-1}(\psi) \geq 0
$$

Therefore for $\delta=\delta(\sigma)$ sufficiently small, as in (3.31),

$$
\left|a_{-n-1}\left(u_{k}\right)\right|+\left|a_{-n-1}(\psi)\right|<\sigma .
$$

For $N=N(k)$ near $\infty$, by $(2.13)$ again,

$$
\left\|u_{k}-v\right\|_{W^{1,2}\left(\Omega_{-N}\right)} \leq \delta
$$

Hence

$$
\left|a_{-N}\left(u_{k}\right)\right|+\left|a_{-N}(\psi)\right|<\sigma .
$$

Finally as in $(3.32,3.44,3.45)$ imply

$$
T_{n}^{-}\left(u_{k}\right)=J_{-N,-n-1}\left(u_{k}\right)+T_{N}^{-}\left(u_{k}\right) \geq-2 \sigma+T_{N}^{-}\left(u_{k}\right) .
$$

Since $J^{*}\left(u_{k}\right)<\infty$, for $N$ possibly still larger,

$$
\left|T_{\mathbb{N}}^{-}\left(u_{k}\right)\right|<\sigma .
$$

Thus $(3.40)$ is a consequence of $(3.48,3.49)$ and $J^{*}(U)=c^{*}$.

It remains to verify (3.42). Since $u_{k} \rightarrow U$ in $L^{2}\left(\Omega_{-n-1}\right)$ as $k \rightarrow \infty$, it suffices to show

$$
\left\|\nabla u_{k}-\nabla U\right\|_{L^{2}\left(\Omega_{-n-1}\right)} \leq \delta / 2
$$

for $n(\delta)$ large and appropriate $k$. Since by weak lower semicontinuity,

$$
\begin{aligned}
& \int_{\Omega_{-n-1}} L(U) \mathrm{d} x \leq \varliminf_{k \rightarrow \infty} \int_{\Omega_{-n-1}} L\left(u_{k}\right) \mathrm{d} x, \\
& 0 \leq \rho_{n}=\underline{\lim }_{k \rightarrow \infty} \int_{\Omega_{-n-1}} L\left(u_{k}\right) \mathrm{d} x-\int_{\Omega_{-n-1}} L(U) \mathrm{d} x .
\end{aligned}
$$

Now (3.50) is a consequence of the following result:

Lemma 3.1. $\rho_{n} \rightarrow 0$ as $n \rightarrow \infty$ and

$$
\underline{\lim }_{k \rightarrow \infty}\left\|\nabla\left(u_{k}-U\right)\right\|_{L^{2}\left(\Omega_{-n-1}\right)}^{2} \leq 2 \rho_{n} .
$$

Proof.

$$
\begin{aligned}
\varliminf_{k \rightarrow \infty} \int_{\Omega_{-n-1}}\left|\nabla\left(u_{k}-U\right)\right|^{2} \mathrm{~d} x & =\varliminf_{k \rightarrow \infty} \int_{\Omega_{-n-1}}\left(\left|\nabla u_{k}\right|^{2}-2 \nabla u_{k} \cdot \nabla U+|\nabla U|^{2}\right) \mathrm{d} x \\
& =\varliminf_{k \rightarrow \infty} \int_{\Omega_{-n-1}}\left|\nabla u_{k}\right|^{2} \mathrm{~d} x-\int_{\Omega_{-n-1}}|\nabla U|^{2} \mathrm{~d} x .
\end{aligned}
$$


Thus to get (3.52), it suffices to prove

$$
\varliminf_{k \rightarrow \infty} \int_{\Omega_{-n-1}}\left|\nabla u_{k}\right|^{2} \mathrm{~d} x \leq \int_{\Omega_{-n-1}}|\nabla U|^{2} \mathrm{~d} x+2 \rho_{n} .
$$

But

$$
\varliminf_{k \rightarrow \infty} \int_{\Omega_{-n-1}} L\left(u_{k}\right) \mathrm{d} x=\int_{\Omega_{-n-1}} L(U) \mathrm{d} x+\rho_{n}=\varliminf_{k \rightarrow \infty} \int_{\Omega_{-n-1}} \frac{1}{2}\left|\nabla u_{k}\right|^{2} \mathrm{~d} x+\int_{\Omega_{-n-1}} G(x, U) \mathrm{d} x
$$

so (3.53) holds. Finally to show that $\rho_{n} \rightarrow 0$ as $n \rightarrow \infty$, by $(2.11,2.12)$ and $(2.16)$

$$
-K \leq J_{-j,-\ell}(U)=\sum_{i=-j}^{-\ell}\left(\varliminf_{k \rightarrow \infty} a_{i}\left(u_{k}\right)-\rho_{-i}\right) \leq \varliminf_{k \rightarrow \infty} J_{-j,-\ell}\left(u_{k}\right)-\sum_{-j}^{-\ell} \rho_{-i} \leq M+2 K-\sum_{-j}^{-\ell} \rho_{-i} .
$$

Thus letting $\ell=0$ and $j \rightarrow-\infty$ shows

$$
\sum_{0}^{\infty} \rho_{n} \leq M+3 K
$$

so $\rho_{n} \rightarrow 0$ as $n \rightarrow \infty$.

Proof of Proposition 2.8. Set

$$
c\left(B_{r}(z)\right)=\inf _{u \in A_{r}(z)} F_{r}(u) .
$$

Since $A_{r}(z)$ is closed and convex, it is weakly closed. The functional $F_{r}$ is weakly lower semicontinuous. Hence there is a $V \in A_{r}$ such that $F_{r}(V)=c\left(B_{r}(z)\right)$. Standard regularity arguments show $V$ is a classical solution of (PDE) and even in $C^{2, \alpha}\left(B_{r}(z)\right)$ for any $\alpha \in(0,1)$. Let

$$
\mathcal{M}\left(B_{r}(z)\right)=\left\{W \in A_{r}(z) \mid F_{r}(W)=c\left(B_{r}(z)\right)\right\} .
$$

Then $\mathcal{M}\left(B_{r}(z)\right)$ is an ordered set via the proof of Proposition 2.1.

We claim each $V \in \mathcal{M}\left(B_{r}(z)\right)$ satisfies $v \leq V \leq w$. If not, suppose $V(\bar{x})>w(\bar{x})$ for some $\bar{x} \in B_{r}(z)$. Set

$$
B=\left\{x \in B_{r}(z) \mid V(x)>w(x)\right\}
$$

Then $\varphi \equiv \min (w, V) \in A_{r}(z)$ so

$$
F_{r}(V) \leq F_{r}(\varphi)
$$

and therefore

$$
\int_{B} L(V) \mathrm{d} x \leq \int_{B} L(w) \mathrm{d} x
$$

For $x \in \bar{\Omega}$ and $j \in \mathbb{Z}$, set $x_{j}=x+(j, 0)$. Define

$$
\left\{\begin{aligned}
\psi(x) & =\max (w(x), V(x)), & & x \in B_{2 r}(z) \\
\psi\left(x_{j}\right) & =\psi(x), & & x \in B_{2 r}\left(z_{j}\right), j \in \mathbb{Z} \\
\psi(x) & =w(x), & & x \in \bar{\Omega} \backslash \bigcup_{j \in \mathbb{Z}} B_{r}\left(z_{j}\right)
\end{aligned}\right.
$$


Then $\psi \in E_{0}$, so

$$
I_{0}(\psi) \geq I_{0}(w)
$$

Hence

$$
\int_{B} L(V) \mathrm{d} x \geq \int_{B} L(w) \mathrm{d} x
$$

Thus by (3.58) and (3.61),

$$
\int_{B} L(V) \mathrm{d} x=\int_{B} L(w) \mathrm{d} x
$$

and

$$
I_{0}(V)=I_{0}(w)=c_{0}
$$

Consequently by (3.59) and (3.62), $\psi \in \mathcal{M}$. But $\psi$ and $w$ are not ordered, contrary to Proposition 2.1. Thus $V \leq w$ and similarly, $V \geq v$.

It remains to show that $F_{r}(U)=c\left(B_{r}(z)\right)$. For $j \in \mathbb{Z}$, let

$$
V_{j}(x)=\inf _{V \in \mathcal{M}\left(B_{r}\left(z_{j}\right)\right)} V(x) .
$$

Then $V_{j}(x) \in \mathcal{M}\left(B_{r}\left(z_{j}\right)\right)$. Define

$$
U^{*}(x)= \begin{cases}V_{j}(x), & x \in B_{r}\left(z_{j}\right) \\ U(x), & x \in \Omega \backslash \bigcup_{j \in \mathbb{Z}} B_{r}\left(z_{j}\right) .\end{cases}
$$

We claim

$$
U^{*} \leq \tau_{-1} U^{*}
$$

If so, $U^{*} \in \Gamma^{*}$ and

$$
J^{*}(U) \leq J^{*}\left(U^{*}\right)
$$

Now (3.64) and (3.66) imply

$$
\int_{B_{r}(z)} L(U) \mathrm{d} x \leq \int_{B_{r}(z)} L\left(V_{0}\right) \mathrm{d} x .
$$

Hence by the minimization property of $V_{0}$,

$$
\int_{B_{r}(z)} L(U) \mathrm{d} x=\int_{B_{r}(z)} L\left(V_{0}\right)=c\left(B_{r}(z)\right) .
$$

Lastly to verify (3.65), suppose it is false. Then for some $j \in \mathbb{Z}$, there is an $(x, y) \in B_{r}\left(z_{j}\right)$ such that

$$
V_{j}\left(x_{0}, y_{0}\right)>V_{j+1}\left(x_{0}+1, y_{0}\right)
$$


Define functions on $B_{2 r}\left(z_{j}\right)$ as follows: $\psi(x, y)=V_{j+1}\left(x_{1}, y\right), \varphi=V_{j}, \chi=\max (\varphi, \psi), \zeta=\min (\varphi, \psi)$. Then on $B_{2 r}\left(z_{j}\right) \backslash B_{r}\left(z_{j}\right)$,

and

$$
\zeta=\varphi=U \leq \tau_{-1} U=\psi=\chi
$$

As in (2.1),

$$
\zeta \in A_{r}\left(z_{j}\right), \quad \tau_{1} \chi \in A_{r}\left(z_{j+1}\right) .
$$

$$
F_{r}(\delta)+F_{r}(\chi)=F_{r}(\varphi)+F_{r}(\psi)=c\left(B_{r}\left(z_{j}\right)\right)+c\left(B_{r}\left(z_{j+1}\right)\right)
$$

which implies that $\zeta \in \mathcal{M}\left(B_{r}\left(z_{j}\right)\right)$ and $\tau_{1} \chi \in \mathcal{M}\left(B_{r}\left(z_{j+1}\right)\right)$. Thus $\zeta \geq \varphi=V_{j}$ and in particular at $\left(x_{0}, y_{0}\right)$,

$$
V_{j}\left(x_{0}, y_{0}\right) \leq \zeta\left(x_{0}, y_{0}\right) \leq \psi\left(x_{0}, y_{0}\right)=V_{j+1}\left(x_{0}+1, y_{0}\right) .
$$

But (3.71) contradicts (3.69). Hence (3.65) holds and Proposition 2.8 is proved.

Proof of Proposition 2.9. The proof here follows the same lines as that of Proposition 2.8 and will be omitted.

\section{FinAL REMARKS}

The results of Section 2 extend to other situations such as those treated in Section 3 of [6] provided that some basic properties of $\mathcal{M}$ carry over to these new settings. In particular it is required that: (i) $\mathcal{M}$ has at least two members so that heteroclinics are possible, (ii) $\mathcal{M}$ is an ordered set, and (iii) $\mathcal{M}$ is not too big, i.e. $\left({ }^{*}\right)$ holds. It will briefly be indicated why this is the case for a class of equations which arise in a water wave model and treated by Kirchgässner [3] near a bifurcation point using center manifold methods.

Consider

$$
-\Delta u=\lambda a(y) u-f(x, y, u, \lambda)
$$

for $(x, y) \in \mathbb{R}^{2}$ with $|y|<1$ with the Dirichlet boundary conditions:

$$
u(x, \pm 1)=0 .
$$

It is assumed that $a(y)$ is $C^{1}$ and positive, $f$ is $C^{1}$ in its arguments, 1-periodic in $x$, and $f(x, y, z, \lambda)=o(|z|)$ as $z \rightarrow 0$. Then the linearization of (4.1) about $u=0$ gives a linear eigenvalue problem:

$$
-\Delta \varphi=\lambda a \varphi
$$

with boundary conditions (4.2) and $\varphi$ 1-periodic in $x$. The smallest eigenvalue $\lambda_{1}$ is positive and simple and there is a corresponding positive eigenfunction $\varphi_{1}$. Choose $\lambda>\lambda_{1}$. Then - see e.g. [6] -

$$
I_{0}(u)=\int_{\Omega_{0}}\left(\frac{1}{2}|\nabla u|^{2}-\frac{\lambda}{2} u^{2}\right) \mathrm{d} x+o\left(\|u\|_{W^{1,2}\left(\Omega_{0}\right)}^{2}\right)
$$

as $u \rightarrow 0$. Since

equation (4.4) shows that if $u$ is a small multiple of $\varphi_{1}$,

$$
\int_{\Omega_{0}}\left|\nabla \varphi_{1}\right|^{2} \mathrm{~d} x=\lambda_{1} \int_{\Omega_{0}} \varphi_{1}^{2} \mathrm{~d} x
$$

$$
I_{0}(u)=\frac{1}{2}\left(1-\frac{\lambda}{\lambda_{1}}\right) \int_{\Omega_{0}}|\nabla u|^{2} \mathrm{~d} x+o\left(\|\nabla u\|_{L^{2} \Omega_{2}}^{2}\right)<0 .
$$


Consequently $c_{0}=c_{0}(\lambda)<0$ for $\lambda>\lambda_{1}$. Of course it is possible that $c_{0}(\lambda)=-\infty$. Some conditions were given in [6] where this is not the case. E.g. $c_{0}(\lambda)>-\infty$ if the primitive, $F(x, y, z, \lambda)=\int_{0}^{z} f(x, y, t, \lambda) \mathrm{d} t$ of $f$ is a bounded function of $z$ or if $F(x, y, z, \lambda) \rightarrow \infty$ as $|z| \rightarrow \infty$.

Assuming that $c_{0}(\lambda)$ is finite, suppose further that $f$ is odd in $z$. Then $F$ is even in $z$. Hence $u \in \mathcal{M}$ implies $-u \in \mathcal{M}$ and by (4.5), $0 \notin \mathcal{M}$. Furthermore the argument of Proposition 2.1 shows $\mathcal{M}$ is an ordered set. Therefore if $v,-v \in \mathcal{M}$, without loss of generality, $-v<0<v$ in $\Omega_{0}$. Consequently our requirements (i-iii) hold here. In particular if $v$ is the smallest positive member of $\mathcal{M}$, there is a pair of solutions of (4.1, 4.2) heteroclinic in $x$, one from $-v$ to $v$ and the other from $v$ to $-v$.

\section{REFERENCES}

[1] V. Bangert, On minimal laminations of the torus. Ann. Inst. H. Poincaré Anal. Non Linéaire 6 (1989) 95-138.

[2] E. Bosetto and E. Serra, A variational approach to chaotic dynamics in periodically forced nonlinear oscillators. Ann. Inst. H. Poincaré Anal. Non Linéaire 17 (2000) 673-709.

[3] K. Kirchgässner, Wave-solutions of reversible systems and applications. J. Differential Equations 45 (1982) 113-127.

[4] A. Mielke, Reduction of quasilinear elliptic equations in cylindrical domains with applications. Math. Mech. Appl. Sci. 10 (1988) 51-66.

[5] J.K. Moser, Minimal solutions of variational problems on a torus. Ann. Inst. H. Poincaré Anal. Non Linéaire 3 (1986) $229-272$.

[6] P.H. Rabinowitz, Solutions of heteroclinic type for some classes of semilinear elliptic partial differential equations. J. Fac. Sci. Univ. Tokyo 1 (1994) 525-550.

[7] P.H. Rabinowitz and E. Stredulinsky, Mixed states for an Allen-Cahn type equation. Comm. Pure Appl. Math. (to appear).

[8] R.E.L. Turner, Internal waves in fluids with rapidly varying density. Ann. Scuola Norm. Sup. Pisa Cl. Sci. Ser. 48 (1981) 513-573. 